\title{
Self-generated electronic heterogeneity and quantum glassiness in the high temperature superconductors
}

\author{
C. Panagopoulos \\ Cavendish Laboratory and IRC in Superconductivity, \\ University of Cambridge, Cambridge CB3 OHE, United Kingdom \\ V. Dobrosavljević \\ Department of Physics and National High Magnetic Field Laboratory, Florida State University, Tallahassee, FL 32306
}

\begin{abstract}
We present a systematic study of the spin and charge dynamics of copper oxide superconductors as a function of carrier concentration $x$. Our results portray a coherent physical picture, which reveals a quantum critical point at optimum doping $\left(x=x_{o p t}\right)$, and the formation of an inhomogeneous glassy state at $x<x_{\text {opt }}$. This mechanism is argued to arise as an intrinsic property of doped Mott insulators, and therefore to be largely independent of material quality and level of disorder.
\end{abstract}

Many interesting materials ranging from magnetorestrictive manganite films [1] and field-effect transistors [2, 3], to unconventional low dimensional superconductors [4], find themselves close to the metal-insulator transition. In this regime, competition between several distinct ground states [4] produces unusual behavior, displaying striking similarities in a number of different systems. Electronic heterogeneity 1, 5] emerges, giving rise to "mesoscopic" coexistence of different ordered phases. Typically, a large number of possible configurations of these local regions have comparable energies, resulting in slow relaxation, aging, and other signatures of glassy systems. Because the stability of such ordering is controlled by doping-dependent quantum fluctuations [ 6,7$]$ introduced by itinerant carriers, these systems can be regarded as prototypical quantum glasses - a new paradigm of strongly correlated matter.

In this Letter we report the emergence and evolution of dynamical heterogeneity and glassy behavior across the phase diagram of the high-transition-temperature $\left(T_{c}\right)$ superconductors (HTS). Based on data of the spin and charge dynamics, we draw a phase diagram (Fig. 1) and propose that self generated glassiness [5] may be a key feature necessary to understand many of the unconventional properties of both the superconducting and the normal state.

Glassiness in the pseudo-gap phase. In the archetypal HTS, $\mathrm{La}_{2-x} \mathrm{Sr}_{x} \mathrm{CuO}_{4}$ (LSCO) the parent 2D antiferromagnetic insulator (AFI) $\mathrm{La}_{2} \mathrm{CuO}_{4}$ displays a sharp peak in the magnetic susceptibility at the Neel temperature $T_{N}=300 \mathrm{~K} . T_{N}$ decreases with hole-doping and the transition width broadens (Fig. 2 - upper panel). Concurrently, there is systematic experimental evidence from various techniques and on several HTS that a second freezing transition $\left(T_{F}\right)$ emerges at lower temperatures with the first added holes (Fig. 2) [8, 9, 10, 11, 12, 13]. At $x>0.02, T_{N}=0$ but the short range order persists [14]: the low-field susceptibility displays a cusp at low temperatures and a thermal hysteresis below, characteristic of a spin glass transition $\left(T_{g}\right)$ (Fig. 2 - upper panel). At $T<T_{g}$ the material displays memory effects like "traditional" spin glasses and is described by an Edwards-Anderson order parameter 15. Interestingly, it is at this doping range that a pseudogap phase develops [4, 16].

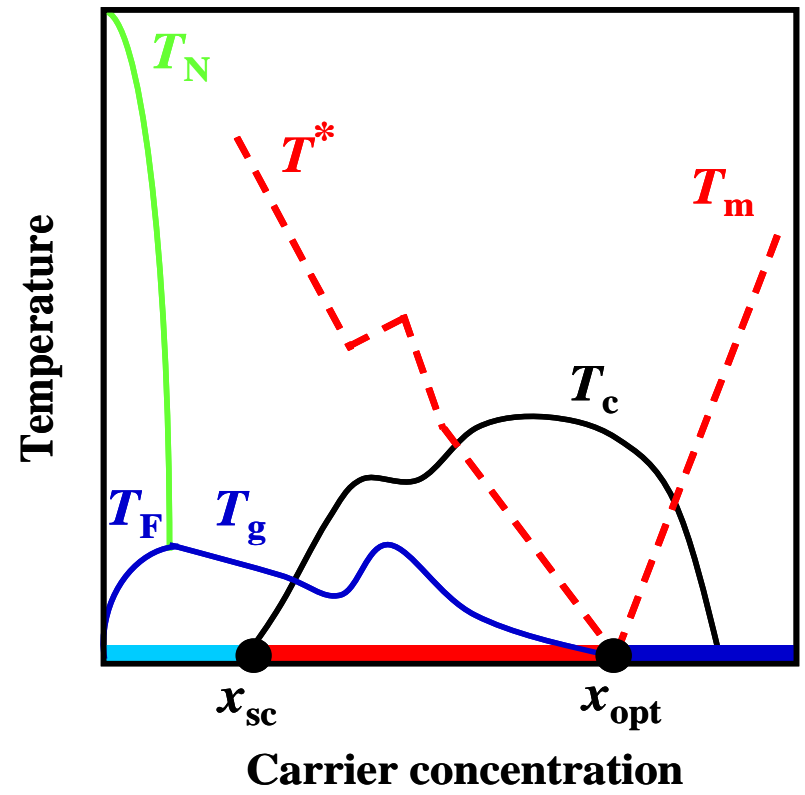

FIG. 1: Schematic plot indicating the three major ground state regimes in the phase diagram of the archetypal HTS. $T_{N}$ is the Neel temperature, $T_{F}$ and $T_{g}$ the onset of short range freezing to an electronic glass, and $T_{c}$ the superconducting transition temperature. At $x<x_{s c}$ the material is a glassy insulator. At $x_{s c}<x<x_{o p t}$ a microscopically inhomogeneous conducting glassy state emerges, with intercalated superconducting and magnetic regions. At $x=x_{o p t}$ the system experiences a quantum glass transition and at $x>x_{\text {opt }}$ the material transforms into a homogeneous metal with BCS-like superconducting properties. The superfluid density is maximum at $x=x_{o p t}$. The crossover scales $T^{*}$ and $T_{m}$ characterizing normal-state transport (see text for details), vanish at the quantum glass transition. 
The emergence of electronic heterogeneity with charge doping is not unique to HTS. It has been demonstrated in semiconductors 3], ruthanates 17], nickelates 18], other copper oxides [18, 19], and manganites [20], where transport experiments indicate that at least some glassy features originate from slow charge dynamics. Further evidence supporting that glassiness in the charge and the spin channels emerge hand-in-hand was recently provided by measurements of the dielectric constant on $\mathrm{La}_{2} \mathrm{Cu}_{1-x} \mathrm{Li}_{x} \mathrm{O}_{4}$ and $\mathrm{La}_{2-x} \mathrm{Sr}_{x} \mathrm{NiO}_{4}$ [18]. These materials are not superconducting, but their spin response is almost identical to that of cuprate superconductors while the dielectric response is remarkably similar to conventional (structural) glasses.

The glassy signatures in the spin channel of HTS suggest analogous effects in the charge response, which we examine by measuring the in-plane electrical resistivity, $\rho_{a b}$, of LSCO single crystals (Fig. 2 - lower panel, inset). The data for $x=0.01-0.04$ show a crossover (resistivity minima) in $\rho_{a b}(T)$ from metallic to insulating-like at a characteristic temperature $T^{*}$ (Fig. 2 - upper panel). Although the crossover takes place over a wide temperature region it clearly occurs at $T \ll T_{N}$ for $x<0.02$ and has a doping dependence similar to $T_{F}$ and $T_{g}$. This similarity indicates the association between short range order and charge retardation. Moreover, the emergence of a glass order with the first added carriers speaks against impurity effects but instead for an intrinsic property.

Coexistence of glassiness and superconductivity. In light of many unconventional properties of HTS below optimum doping [4], it is important to probe the possible correlation between the identified dynamical heterogeneities and superconductivity (present for $x>x_{s c}=$ 0.05 for LSCO). Muon spin relaxation ( $\mu \mathrm{SR})$ has been successful in identifying the freezing of electronic moments under the superconducting dome of various HTS 8, 9, 11, 14, 21, 22]. Figure 3 (inset) shows a typical example (LSCO, $x=0.08\left(T_{c}=21 \mathrm{~K}\right)$ ) of spectra with a glass transition at low temperatures displaying an initial rapid relaxation. The amplitude of the muon spin polarization reveals that all muons inside the sample experience a non-zero local field indicating that the magnetism persists throughout the entire volume of the sample. However, the absence of a dip in the polarization function at the lowest times indicates the presence of a large number of low field sites - superconducting and magnetic regions intercalated on a microscopic ( $\leq 2 \mathrm{~nm})$ scale $8,9,14,21]$. These observations suggest the presence of magnetic stripes/droplets, in agreement with independent indications [4, 8, 9, 10, 11, 13, 14, 21] from transport and spectroscopic studies for the nonsuperconducting dopings. From these studies we may conclude that superconductivity coexists with glassiness on a microscopic scale throughout the bulk of the material. This behavior is not limited to LSCO - although the latter has been investigated most extensively. Simi-
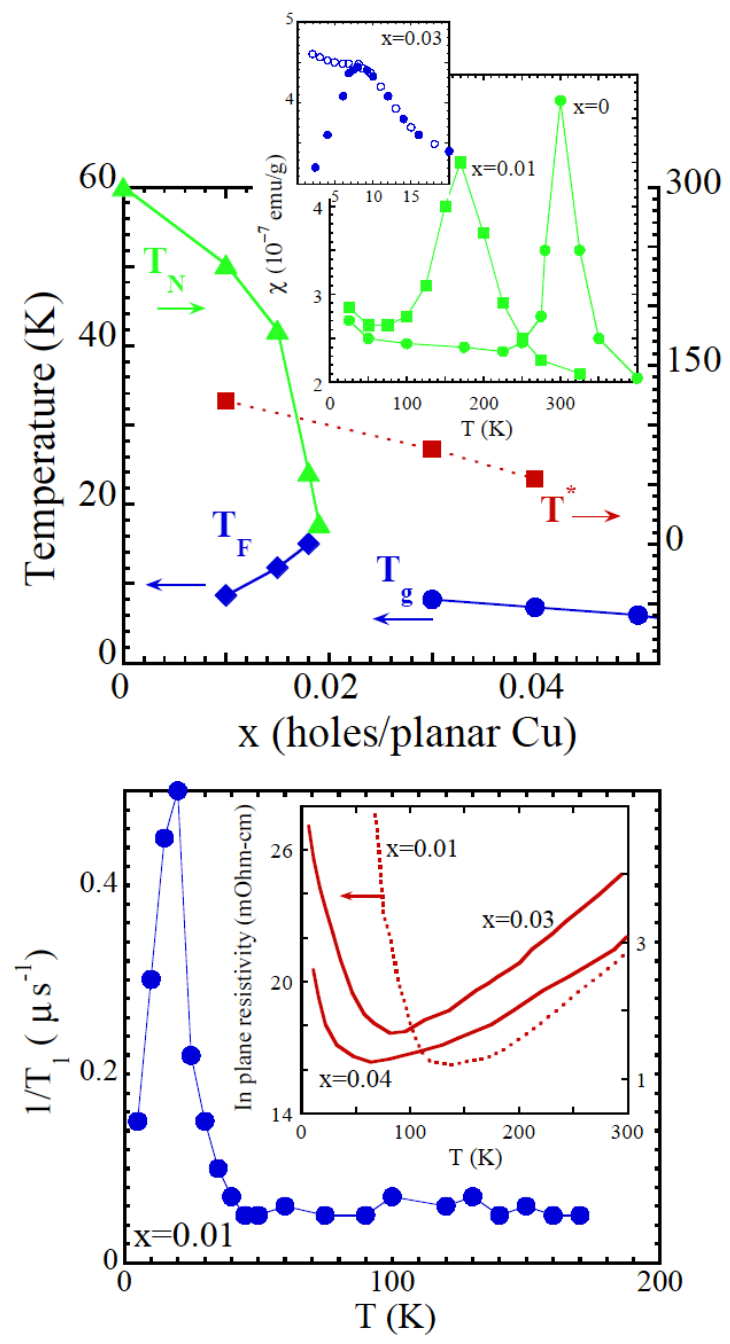

FIG. 2: The upper panel depicts the doping dependence of the Neel temperature $T_{N}$, the second freezing $T_{F}$ and glass temperature $T_{g}$. The latter two scales are determined by a peak in the spin lattice relaxation $1 / T_{1}$ (see e.g., lower panel) - data obtained by zero field $\mu S R$ on LSCO $(x=0.01)$. $T^{*}$ is a crossover from metallic-like to insulating-like resistivity determined from the in-plane resistivity measurements shown as inset to the lower panel. The inset in the upper panel depicts the temperature dependence of the susceptibility for LSCO $(x=0,0.01)$ single crystals with $H / / c$. The associated inset is data for $x=0.03$ showing the transformation of the material to a glass.

lar results have been observed in the "stripe-compound" Nd-LSCO, pure and $\mathrm{Y}$ doped Bi-Sr-Ca-Cu-O, pure or $\mathrm{Ca}$ doped $\mathrm{Y}-\mathrm{Ba}-\mathrm{Cu}-\mathrm{O}$ and more recently $\mathrm{Ca}-\mathrm{Na}-\mathrm{Cu}-\mathrm{O}-$ $\mathrm{Cl}$ 8, 9, 10, 11, 13, 14, 21.

Spin-charge correlations around the $S C$ dome. To further examine the region where $T_{c}>0$, we re-analyzed early measurements in high magnetic fields [23] where bulk superconductivity was suppressed, revealing information about low- $T$ charge transport in the normal 


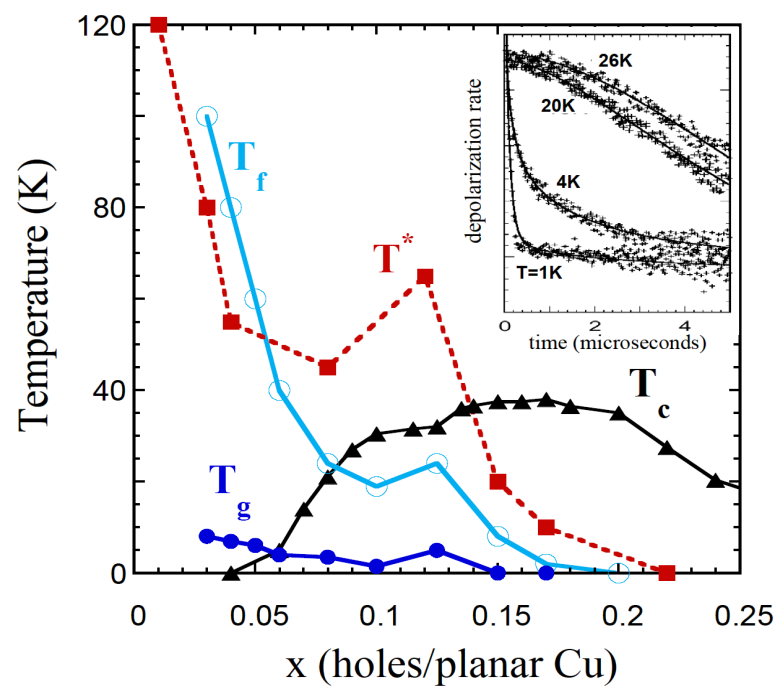

FIG. 3: Doping dependence of the superconducting transition temperature $T_{c}$, the onset of slowing down of electronic moments, $T_{f}$, before being frozen at the glass temperature $T_{g} . T^{*}$ is a crossover from metallic-like resisitivity to insulating-like. The inset depicts $\mu S R$ spectra for $x=0.08$ at different temperatures, showing a deviation from a Gaussian signalling the entrance of fluctuating electronic moments into the $\mu S R$ frequency window at approximately $25 \mathrm{~K}\left(=T_{f}\right)$ and their eventual freezing below $4 \mathrm{~K}\left(=T_{g}\right)$.

phase. A striking similarity is observed between the doping dependence of the spin freezing and the resistivity minima all the way to the overdoped region (Fig. 3). Both $T_{g}$ and $T^{*}$ decrease upon doping, except for an increase around $x=1 / 8$ (Fig. 3), which is thought to reflect stripe pinning 4 , or some other form of commensurate charge ordering [24]. Furthermore, the doping dependence of the resistivity minima closely tracks the onset of the slowing down of spin fluctuations $\left(T=T_{f}\right)$, before they freeze into a glassy state $\left(T=T_{g}\right)$. This observation is consistent with theoretical predictions [6] that the effective disorder

$$
W_{e f f}=\left[W^{2}+V^{2} q_{E A}\right]^{1 / 2}
$$

seen by the charge carriers can be strongly enhanced by static and dynamic fluctuations associated with glassy ordering. Here, $W$ is the energy scale of the impurity potential, $V$ measures the electron-electron interactions, and $q_{E A}$ is the frozen order parameter fluctuation in the glassy phase [6]. Assuming that the selfgenerated randomness dominates the impurity potential (i.e. $\left.W^{2} \ll V^{2} q_{E A}\right)$, this mechanism also explains the correlations between the doping dependence of $T_{g}$ and $T^{*}$. This is true since one expects $T_{g}(x) \sim\left[q_{E A}(x)\right]^{1 / 2}$, and the resistivity crossover scale $T^{*}$ should be set by the effective disorder $W_{\text {eff }}$.
Intermediate conducting glass phase. The correlation between conductivity and glassiness indicates that for $x<x_{s c}(=0.05)$ we are dealing with a strongly localized insulator displaying hopping transport at $T<T^{*}$. Here, the number of free carriers can be expected to vanish at $T=0$, in agreement with recent Hall-effect measurements 25]. On the other hand, for $0.05<x<0.20$ the number of carriers is found to be finite [25], suggesting an itinerant system even in the normal phase. In this regime, DC transport has a much weaker 23] (although still insulating-like) temperature dependence. However, the observed $\log T$ resistivity upturn in this region has been shown 23] to be inconsistent with conventional localization/interaction corrections which could indicate an insulating ground state. Instead, estimates 26] reveal this behavior to be consistent with that expected for metallic droplet charging/tunnelling processes, as seen in quantum dots and granular metals 26]. These results suggest that in this regime HTS are inhomogeneous metals, where conducting droplets connect throughout the sample, and a metal-insulator transition in the normal phase happens exactly at $x=x_{s c}$. At lower densities the conducting droplets remain isolated, and the material may be viewed as an insulating cluster or stripe glass. As carrier concentration increases they connect and the carriers are free to move throughout the sample, forming filaments or "rivers". This is, in fact, the point where free carriers emerge in Hall-effect data 25] and phase coherent bulk superconductivity arises at $x>x_{s c}$. This observation indicates that it is the inhomogeneous nature of the underdoped glassy region which controls and limits the extent of the superconducting phase at low doping.

Therefore, based on evidence for charge retardation, freezing, and uniformly distributed electronic heterogeneity in the form of glassy stripes or droplets, we propose that in the interval $x_{s c}<x<x_{o p t}$ a novel intermediate phase arises in the form of a bad metal. The emergence of such an intermediate conducting glass phase separating a conventional metal and a glassy insulator has, in fact, been predicted in recent theoretical work [7].

Quantum glass transition. We now ask whether a true quantum critical point (QCP) separates the glassy nonFermi liquid and the metallic-Fermi liquid-like regimes. We need an experiment where one may gradually increase the amount of disorder, enhance short-range correlations, suppress superconductivity, and fully expose the glassy ground state. These conditions are met by $Z^{2+}$ doping [9, 14, 27]. Figure 4 depicts characteristic data for $\mathrm{La}_{2-x} \mathrm{Sr}_{x} \mathrm{Cu}_{0.95} \mathrm{Zn}_{0.05} \mathrm{O}_{4}$ with the normal state exposed $\left(T_{c}=0\right)$ across the $T-x$ phase diagram. The similar doping dependence of $T_{g}$ for pure and up to $5 \% \mathrm{Zn}$ doped samples [9, 14, 27] indicates that regardless of a sample being pure, disordered (Zn-doped), superconducting or not we obtain universal behavior: A set of glassy phase transitions, enhanced near $x=1 / 8$, and ending at the same doping, supporting the presence of a quan- 
tum glass transition insensitive to the amount of disorder. These results strongly suggest that glassiness is not driven by impurities but is predominantly self-generated, consistent with those theoretical scenarios that predict phase separation [4] at low doping. Coulomb interactions, however, enforce charge neutrality and prevent 4] global phase separation; instead, the carriers are expected [5] to segregate into nano-scale domains - to form a stripe/cluster glass [5]. As quantum fluctuations increase upon doping [6, 7], such a glassy phase should be eventually suppressed at a quantum critical point, which in LSCO emerges around $x=x_{\text {opt }} \approx 0.2$. Remarkable independent evidence that a $\mathrm{QCP}$ is found precisely at $x=x_{\text {opt }}$ is provided by the observation of a sharp change in the superfluid density $n_{s}(0) \sim 1 / \lambda_{a b}^{2}(0)$ (where $\lambda_{a b}(0)$ is the absolute value of the in-plane penetration depth). At $x>x_{o p t}, n_{s}(0)$ is mainly doping independent (Fig. 4 ), while the $T$-dependence is in good agreement with the BCS weak-coupling $d$-wave prediction [9]. At dopings below the quantum glass transition $n_{s}(0)$ is rapidly suppressed (note the enhanced depletion near $x=1 / 8$ precisely where $T_{g}$ and $T^{*}$ are enhanced) and there is a marked departure of $n_{s}(T)$ from the canonical weak coupling curve 9]. Similar behavior has been observed in other HTS and in the $c$-axis component [9]. The penetration depth data show that the onset of quasistatic magnetic and charge correlations coincides with an abrupt change in the superconducting ground state. In addition, a crossover temperature $T_{m}$ at $x>x_{\text {opt }}$ separating marginal Fermi liquid transport at $T>T_{m}$ from more conventional metallic behavior at $T<T_{m}$ also seems to drop [28] to very small values around optimum doping (see Fig. 1). At $x>x_{\text {opt }}$ the ground state becomes metallic and homogeneous, with no evidence for glassiness or other form of nano-scale heterogeneity 9, 14, 23, 25, 27, 29]. All these results provide strong evidence of a sharp change in ground state properties at $x=x_{\text {opt }}$, and the emergence of vanishing temperature scales as this point is approached - just as one expects at a QCP. Let us note, the extent of the region between $x_{s c}$ and $x_{o p t}$ is material dependent and expected to vary across the HTS families.

In summary, we have identified three distinct doping regimes: (1) $x<x_{s c}$; (2) $x_{s c}<x<x_{o p t}$; and (3) $x>x_{\text {opt }}$, separated by two critical points: a quantum glass transition at $x=x_{o p t}$ and a normal state metalinsulator transition at $x=x_{s c}$ within the glassy phase. On this basis we propose the behavior of HTS could bear resemblance to other materials close to disorder-driven metal-insulator transitions, where electronic heterogeneity and self-generated glassiness arise with the first added holes - a mechanism that may potentially explain many puzzling features of cuprate superconductors.

CP acknowledges earlier collaboration with M. Kodama, E. Liarokapis, T. Nishizaki, N.J. Owen and T. Sasagawa. We thank L. Gor'kov, E. Manousakis, S.

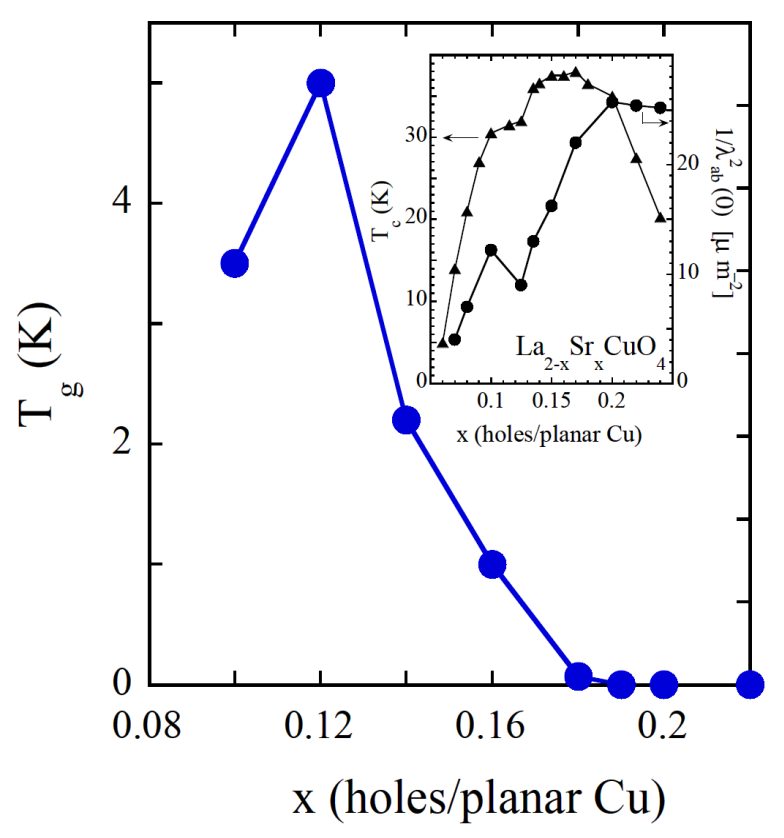

FIG. 4: Doping dependence of the glass transition temperature $T_{g}$ for LSCO doped with $5 \% \mathrm{Zn}$. The inset shows data for the superconducting transition temperature $T_{c}$ and superfluid density $n_{s}(0) \sim 1 / \lambda_{a b}^{2}(0)$ for the pure LSCO system, indicating the transition in the superconducting ground state precisely at the concentration where glassiness vanishes.

Sachdev, and J. Schmalian for helpful discussions. The work in Cambridge was supported by The Royal Society and the ISIS Rutherford Appleton Laboratory. The work at FSU was supported through grant NSF-0234215.

[1] E. Dagotto, Nanoscale Phase Separation and Colossal Magnetoresistance (Springer-Verlag, Berlin, 2002).

[2] E. Abrahams et al., Rev. Mod. Phys. 73, 251 (2001).

[3] S. Bogdanovich and D. Popović, Phys. Rev. Lett. 88, 236401 (2002).

[4] S. A. Kivelson et al., Rev. Mod. Phys. 75, 1201 (2003).

[5] J. Schmalian and P. G. Wolynes, Phys. Rev. Lett. 85, 836 (2000).

[6] A. A. Pastor and V. Dobrosavljević, Phys. Rev. Lett. 83, 4642 (1999).

[7] V. Dobrosavljević et al., Phys. Rev. Lett. 90, 016402 (2003).

[8] C. Niedermayer et al., Phys. Rev. Lett. 80, 3843 (1998).

[9] C. Panagopoulos et al., Solid State Comm. 126, 47 (2003) and references therein.

[10] M. Matsuda et al., Phys. Rev. B, 62, 134515 (2002).

[11] S. Sanna et al., preprint, cond-mat/0403608 (2004).

[12] K. Ishida et al., Phys. Rev. Lett. 92, 257001 (2004).

[13] Y. Kohsaka et al., Phys. Rev. Lett. 93, 097004 (2004).

[14] C. Panagopoulos et al., Phys. Rev. B 66, 064501 (2002).

[15] F. C. Chou et al., Phys. Rev. Lett. 75, 2204 (1995). 
[16] T. Hanaguri et al., Nature (London) 430, 1001 (2004).

[17] S. Nakatsuji et al., Phys. Rev. Lett. 93, 146401 (2004).

[18] T. Park et al., preprint, cond-mat/0404446 (2004).

[19] T. Sasagawa et al., Phys. Rev. B 66, 184512 (2002).

[20] A. Bhattacharya et al., preprint, cond-mat/0407607 (2004).

[21] A. Kanigel et al., Phys. Rev. Lett. 88, 137003 (2002).

[22] T. Hanaguri, Gordon Research Conference (Oxford 2004).
[23] G. S. Boebinger et al., Phys. Rev. Lett. 77, 5417 (1996).

[24] P. W. Anderson (2004), preprint, cond-mat/0406038

[25] F. F. Balakirev et al., Nature 424, 912 (2003).

[26] I. S. Beloborodov et al., Phys. Rev. Lett. 91, 246801 (2003).

[27] C. Panagopoulos et al., Phys. Rev. B 69, 144510 (2004).

[28] S.H. Naqib et al., Physica C 387, 365 (2003).

[29] K. McElroy, et al., preprint, cond-mat/0404005 (2004). 